\title{
Alternative reproductive strategies of Hypocrea orientalis and genetically close but clonal Trichoderma longibrachiatum, both capable of causing invasive mycoses of humans
}

Correspondence

Irina S. Druzhinina

druzhini@mail.zserv.tuwien.ac.at

Received 7 June 2008

Revised 14 July 2008

Accepted 21 July 2008

\author{
Irina S. Druzhinina, ${ }^{1}$ Monika Komoń-Zelazowska, ${ }^{1}$ László Kredics, ${ }^{2}$ \\ Lóránt Hatvani, ${ }^{2}$ Zsuzsanna Antal, ${ }^{2}$ Temesgen Belayneh ${ }^{1}$ \\ and Christian P. Kubicek ${ }^{1}$
}
${ }^{1}$ Research Area of Gene Technology and Applied Biochemistry, Institute of Chemical Engineering, Vienna University of Technology, Getreidemarkt 9/1665, A-1060 Vienna, Austria
${ }^{2}$ Department of Microbiology, Faculty of Science and Informatics, University of Szeged, Közép fasor 52, H-6726 Szeged, Hungary

The common soil fungus Trichoderma (teleomorph Hypocrea, Ascomycota) shows increasing medical importance as an opportunistic human pathogen, particularly in immunocompromised and immunosuppressed patients. Regardless of the disease type and the therapy used, the prognosis for Trichoderma infection is usually poor. Trichoderma longibrachiatum has been identified as the causal agent in the majority of reported Trichoderma mycoses. As T. longibrachiatum is very common in environmental samples from all over the world, the relationship between its clinical and wild strains remains unclear. Here we performed a multilocus (ITS1 and 2, tef1, cal1 and chit185) phylogenetic analysis of all available clinical isolates (15) and 36 wild-type strains of the fungus including several cultures of its putative teleomorph Hypocrea orientalis. The concordance of gene genealogies recognized $T$. longibrachiatum and $H$. orientalis to be different phylogenetic species, which are reproductively isolated from each other. The majority of clinical strains (12) were attributed to $T$. longibrachiatum but three isolates belonged to $H$. orientalis, which broadens the phylogenetic span of human opportunists in the genus. Despite their genetic isolation, $T$. longibrachiatum and $H$. orientalis were shown to be cosmopolitan sympatric species with no bias towards certain geographical locations. The analysis of haplotype association, incongruence of tree topologies and the split decomposition method supported the conclusion that $H$. orientalis is sexually recombining whereas strict clonality prevails in $T$. longibrachiatum. This is a rare case of occurrence of sexual reproduction in opportunistic pathogenic fungi. The discovery of the different reproduction strategies in these two closely related species is medically relevant because it is likely that they would also differ in virulence and/or drug resistance. Genetic identity of environmental and clinical isolates of $T$. longibrachiatum and $H$. orientalis suggests the danger of nosocomial infections by Hypocrea/Trichoderma and highlights the need for ecological studies of spore dispersal as source of invasive human mycoses.

\section{INTRODUCTION}

Many mould species are capable of causing invasive mycoses of mammals, including humans, under appropriate conditions, but of the more than one million fungal species which are estimated to exist in nature, only a few hundred have been associated with human and animal diseases (Walsh \& Groll, 1999). In the last decade, however, case reports on infections by common mould fungi have

Abbreviations: ITS, internal transcribed spacer; MCMC, metropoliscoupled Markov chain Monte Carlo; PHT, partition homogeneity test. increased, due to HIV/AIDS and the use of immunosuppressants for organ transplantation and cancer therapies. Species from the fungal genus HypocrealTrichoderma (Hypocreales, Ascomycota) have recently also joined this emerging list of such opportunistic pathogens. Detailed case reports of Trichoderma infections have been summarized by Kredics et al. (2003). Typically, these include several isolations from the peritoneal effluent of dialysis patients, infections of immunocompromised transplant recipients, and patients suffering from leukaemia, brain abscesses and HIV (Furukawa et al., 1998; Hennequin et al. 2000; Munoz 
et al. 1997; Myoken et al. 2002). While Trichoderma isolates are still not a major threat, they nevertheless pose difficult diagnostic and therapeutic challenges because (i) without rapid diagnosis and treatment their clinical manifestations can be fatal (Seguin et al., 1995; Tanis et al., 1995; Richter et al., 1999; Chouaki et al., 2002; Myoken et al., 2002; Tang et al., 2003), (ii) they are difficult to identify by morphological analysis (Druzhinina et al., 2005), and (iii) they are resistant to most antifungal agents (Kratzer et al., 2006).

The sources of human and animal infections by Trichoderma species, which typically are cosmopolitan soil-borne fungi frequently found in the rhizosphere or as endophytes (Klein \& Eveleigh 1998; Harman et al., 2004), are not known at present. Sequence analysis of the internal transcribed spacers 1 and 2 (ITS1 and 2) of the rRNA gene cluster identified all strains isolated from clinical patients with a few exceptions - as Trichoderma longibrachiatum (Kuhls et al., 1999; Kredics et al., 2003). This taxon usually represents a common, albeit minor, component of Trichoderma communities isolated from soil and other environments (Druzhinina et al., 2005; Kubicek et al., 2003; Kullnig et al., 2000; Wuczkowski et al., 2003; Zhang et al., 2005 ,), but it appears to be more abundant in indoor environments such as water-damaged buildings or mushroom farms infected by green mould disease (Thrane et al., 2001; Hatvani et al., 2007, respectively). Consequently, $T$. longibrachiatum has also been detected in sputum and sinus ethmoidalis of healthy humans (Kredics et al., 2003).

Many facultative pathogenic fungi such as Trichophyton rubrum, Cryptococcus neoformans and the pathogenic chytrid Batrachochytrium dendrobatidis have been shown to be single worldwide distributed clonal lineages (Gräser et al., 1999; Halliday \& Carter, 2003; Morehouse et al., 2003, Zhang et al., 2006). However, some other opportunistic human pathogenic fungi such as Aspergillus fumigatus exhibit both clonal and recombining history (Nielsen \& Heitman, 2007; Pringle et al., 2005). The reproduction strategy and population structure of $T$. longibrachiatum has not been investigated yet. Phylogenetic analyses of Trichoderma section Longibrachiatum has been limited to the sequence of the ITS regions of the rRNA genes (Kuhls et al., 1997) and RAPD (random amplified polymorphic DNA) fingerprinting (Turner et al., 1997), which both yielded results suggesting clonality for T. longibrachiatum. However, as the ascomycete Hypocrea orientalis has been proposed as a teleomorph of T. longibrachiatum (Samuels et al., 1998), at least some generations of sexual reproduction should thus be detectable in its population history.

Knowledge of the population structures of T. longibrachiatum and $H$. orientalis, their relationship and mode(s) of reproduction would therefore aid our understanding of whether clinical infections are caused by certain lineages only (i.e. whether they share the same recent ancestry), and whether the causative agents are present in all or only some geographical areas. The objectives of this study were therefore to (i) investigate the phylogenetic relatedness of a geographically broad sample of $T$. longibrachiatum and $H$. orientalis including clinical and environmental strains; (ii) to detect the genetic origins of clinical isolates; and (iii) to identify the mode of reproduction of these fungi with special emphasis on that of the clinical isolates.

\section{METHODS}

Material studied. The strains, their origin and the sequences used in this work are listed in Table 1 . The isolates are stored at $-80{ }^{\circ} \mathrm{C}$ in $50 \%$ glycerol in the laboratory of Vienna University of Technology (TU Wien). Strains are grouped according to their identification in the present work. For convenience, TU Wien collection codes (C.P.K.) are used for the strains throughout the work, but other collection numbers are also listed in Table 1.

DNA extraction, PCR amplification and sequencing. Mycelia were harvested after 2-4 days growth on 3\% malt extract agar (MEA) at $25{ }^{\circ} \mathrm{C}$ and genomic DNA was extracted using the Qiagen DNeasy Plant Maxi kit following the manufacturer's protocol. Amplification of the nuclear rRNA gene cluster, containing ITS1 and 2 and the 5.8S rRNA gene, and of a fragment of the endochitinase gene chil8-5 (formerly named ech42) was done as described previously (KomońZelazowska et al., 2007). The fourth large intron of tef1 (translation elongation factor $1-\alpha$ ) was amplified using primers EF1-728F (5'CATCGAGAAGTTCGAGAAGG-3') and TEF1-LLErev (5'-AACTTGCAGGCAATGTGG-3') (Jaklitsch et al., 2006), and a fragment of call (calmodulin) using primers CAL-228F (5'-GAGTTCAAGGAGGCCTTCTCCC-3') and CAL-737R (5'-CATCTTTCTGGCCATCATGG3') (Chaverri et al., 2003). Purified PCR products for ITS1 and 2, tefl, call and chit18-5 were subjected to automatic sequencing at MWG (Martinsried, Germany). NCBI GenBank accession numbers of the corresponding sequences are given in Table 1.

Sequence analysis. DNA sequences were aligned with CLUSTAL X 1.81 (Thompson et al., 1997) and then visually edited using GeneDoc 2.6 (Nicholas \& Nicholas, 1997). The possibility of intragenic recombination, which would prohibit the use of the respective loci for phylogenetic analysis, was tested by linkage-disequilibrium-based statistics as implemented in DnaSP 4.50 .3 (Rozas et al., 2003). The neutral evolution of coding sequences (call and chil8-5) was tested by Tajima test implemented in the same software. The interleaved NEXUS file was formatted using PAUP*4.0b10 (Swofford, 2002) and manually formatted for the MrBayes v3.2 program (Ronquist \& Huelsenbeck, 2003). The best nucleotide substitution model for each locus was determined using jMODELTEST (Posada, 2008). As Akaike and Bayesian information criteria [AIC (Akaike, 1974) and BIC (Schwarz, 1978), respectively] selected different nucleotide substitution models for every locus and due to the relatively small size of individual datasets ( 731 characters per 51 sequences for the biggest) the unconstrained GTR $+\mathrm{I}+\mathrm{G}$ substitution model was applied to all sequence fragments (Table 2). Metropolis-coupled Markov chain Monte Carlo (MCMC) sampling was performed with two simultaneous runs of four incrementally heated chains that performed either 1 or 3 million generations. The length of run (number of generations) for each dataset was determined using the AWTY graphical system (Wilgenbusch et al., 2004, online at http://ceb.csit.fsu.edu/awty) to check the convergence of MCMC; all analyses were repeated at least twice. Bayesian posterior probabilities (PP) were obtained from the $50 \%$ majority rule consensus of trees sampled every 100 generations after removing the first trees using the 'burn' command. The number of 'burned' generations was determined for every run based on visual 
Table 1. Clinical and environmental strains of Trichoderma, their origin, and NCBI accession numbers of corresponding sequences

\begin{tabular}{|c|c|c|c|c|c|c|c|c|}
\hline Species & $\begin{array}{l}\text { TU Wien } \\
\text { identifier }\end{array}$ & Alternative strain nos & Substratum & Country & ITS1 and 2 & tef 1 & chi18-5 & cal1 \\
\hline \multirow[t]{33}{*}{$\begin{array}{l}\text { Trichoderma } \\
\text { longibrachiatum }\end{array}$} & $\begin{array}{l}\text { C.P.K. } 1254 \\
\text { ex-type }\end{array}$ & $\begin{array}{l}\text { CBS 818.68, DAOM } \\
167674\end{array}$ & Mud in creek & Ohio, USA & EU401556 & EU401591 & EU401511 & EU401459 \\
\hline & C.P.K. 1685 & UAMH7955 & Human sinus & NA & AY328040 & EU401596 & EU401516 & EU401464 \\
\hline & C.P.K. 1686 & UAMH7956 & $\begin{array}{l}\text { Lung tissue of a bone marrow } \\
\text { transplantation patient }\end{array}$ & NA & AY328041 & EU401597 & EU401517 & EU401465 \\
\hline & C.P.K. 1687 & UAMH 9515 & Peritoneal effluent & $\mathrm{NA}$ & AY328035 & EU401598 & EU401518 & EU401466 \\
\hline & C.P.K. 1689 & ATCC 201044 & Neutropenic child & Texas, USA & AY585879 & EU401600 & EU401520 & EU401468 \\
\hline & C.P.K. 1690 & ATCC 208859 & HIV-positive patient & Texas, USA & AY328042 & EU401601 & EU401521 & EU401469 \\
\hline & C.P.K. 1691 & CBS 446.95 & Lung tissue & Vienna, Austria & AY328039 & EU401602 & EU401522 & EU401470 \\
\hline & C.P.K. 1692 & IP 2110.92 & $\begin{array}{l}\text { Lung and heart of bone marrow } \\
\text { transplantation patient }\end{array}$ & Villejuif, France & Z82902 & EU401603 & EU401523 & EU401471 \\
\hline & C.P.K. 1693 & CNM-CM 382 & Peritoneal fluid of CAPD $\dagger$ patient & Las Palmas, Spain & AY328034 & EU401604 & EU401524 & EU401472 \\
\hline & C.P.K. 1695 & CNM-CM 1798 & $\begin{array}{l}\text { Blood culture of patient with liver } \\
\text { transplant }\end{array}$ & Spain & AY920396 & EU401605 & EU401525 & EU401473 \\
\hline & C.P.K. 1696 & CNM-CM 2171 & $\begin{array}{l}\text { Foot skin of premature infant with } \\
\text { subcutaneous lesions }\end{array}$ & Spain & AY920397 & EU401606 & NA & EU401474 \\
\hline & C.P.K. 1697 & CNM-CM 2277 & Sputum of tuberculosis patient & Spain & AY920398 & EU401607 & EU401526 & EU401475 \\
\hline & C.P.K. 2882 & SzMC IM3 & $\begin{array}{l}\text { Sinus lavage sample of a rhinosinusitis } \\
\text { patient }\end{array}$ & NA & EU401576 & EU401627 & EU401546 & EU401495 \\
\hline & C.P.K. 2879 & SzMC Thg & Water-damaged building & Finland & EU401573 & EU401624 & EU401543 & EU401492 \\
\hline & C.P.K. 1698 & $\begin{array}{l}\text { CNM-CM 1698, IMI } \\
297702\end{array}$ & Biocontrol strain & Spain & AY585880 & EU401608 & EU401527 & EU401476 \\
\hline & C.P.K. 2058 & SzMC 3102, B17 & Agaricus compost & Hungary & $\mathrm{NA}$ & EU401620 & EU401539 & EU401488 \\
\hline & C.P.K. 850 & Y 20, CBS 115341 & Loamy sand, guava plantation & Alexandria, Egypt & EU401555 & EU401590 & EU401510 & EU401458 \\
\hline & C.P.K. 1749 & DAOM 231259 & Sandy soil, parkland & Kigali, Rwanda & EU401562 & EU401611 & EU401530 & EU401479 \\
\hline & C.P.K. 1750 & DAOM 231258 & Sandy soil, parkland & Kigali, Rwanda & EU401563 & EU401612 & EU401531 & EU401480 \\
\hline & C.P.K. 2056 & SzMC 3001, A35 & Pleurotus cultivation substratum & Hungary & EU401569 & EU401619 & EU401538 & EU401487 \\
\hline & C.P.K. 624 & TUB F-363 & Soil & Lisbon, Portugal & EU401551 & EU401582 & EU401501 & EU401449 \\
\hline & C.P.K. 45 & IMI 297702 & Soil & Egypt & NA & EU401578 & EU401497 & EU401445 \\
\hline & C.P.K. 848 & Y 17, IMI 155340 & Clay, under orange tree & El-Mansura, Egypt & EU401553 & EU401588 & EU401508 & EU401456 \\
\hline & C.P.K. 1889 & PPRC S3 & Red soil, coffee-growing region & Ethiopia & EU401567 & EU401617 & EU401536 & EU401485 \\
\hline & C.P.K. 1301 & IMI 287096 & Unknown & India & EU401559 & EU401594 & EU401514 & EU401462 \\
\hline & C.P.K. 1303 & IMI 291914 & Unknown & Antarctica & EU401560 & EU401595 & EU401515 & EU401463 \\
\hline & C.P.K. 2062 & CECT 2412 & Mushroom compost & Wales, UK & EU401572 & EU401623 & EU401542 & EU401491 \\
\hline & C.P.K. 2059 & SzMC 3103, B19 & Agaricus compost & Hungary & EU401570 & EU401621 & EU401540 & EU401489 \\
\hline & C.P.K. 1707 & & Soil & Kazan, Russia & EU401561 & EU401610 & EU401529 & EU401478 \\
\hline & C.P.K. 680 & TUB F-828 & Flower soil & Mexico City, Mexico & AY857222 & EU401583 & EU401502 & EU401450 \\
\hline & C.P.K. 1890 & PPRC S8 & Red soil, coffee-growing region & Ethiopia & EU401568 & EU401618 & EU401537 & EU401486 \\
\hline & C.P.K. 710 & TUB F-1036 & Soil in artificial rain forest & Mexico City, Mexico & AY857241 & EU401586 & EU401506 & EU401454 \\
\hline & C.P.K. 1815 & PPRC J9 & Red soil, coffee-growing region & Jimma, Ethiopia & EU401564 & EU401613 & EU401532 & EU401481 \\
\hline
\end{tabular}


Table 1. cont.

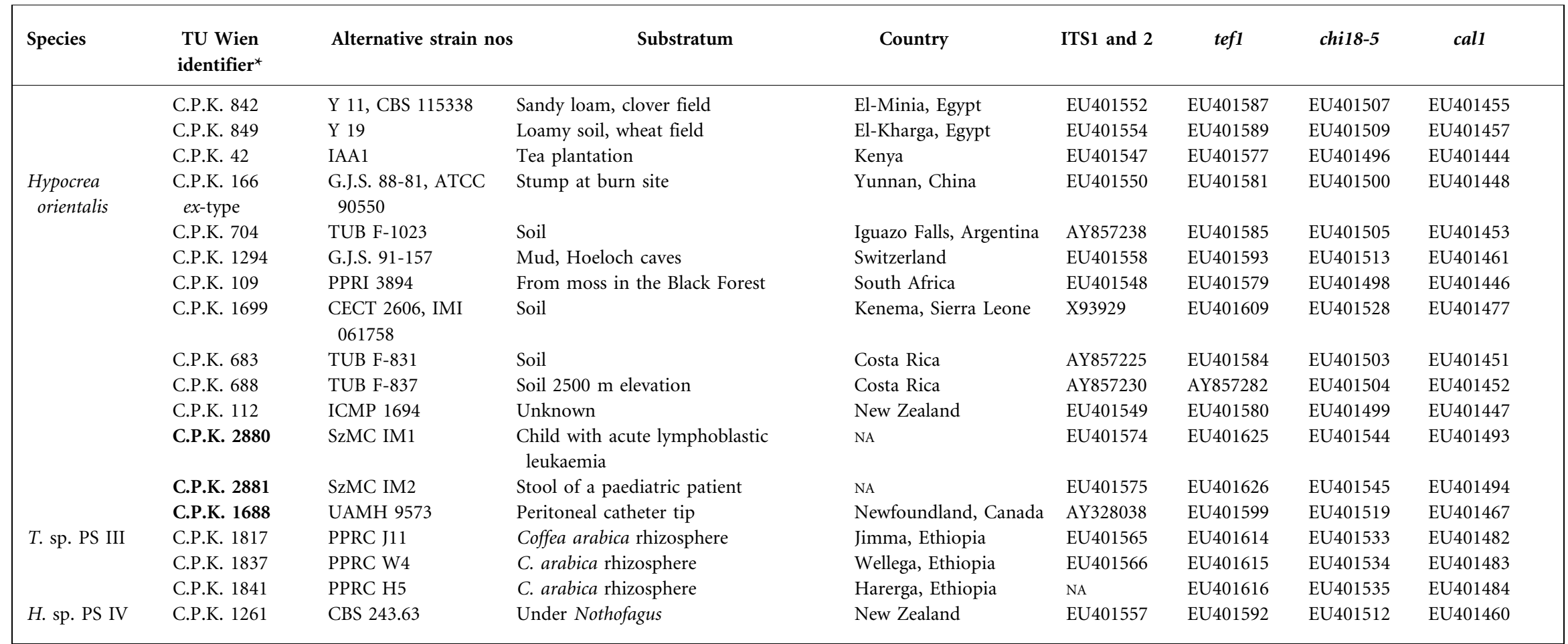

NA, Not available.

${ }^{*}$ Bold type highlights clinical strains.

$\uparrow \mathrm{CAPD}$, continuous ambulatory peritoneal dialysis. 
Table 2. Nucleotide characteristics of loci and parameters of phylogenetic analyses

AIC and BIC, Akaike information criterion (Akaike, 1974) and Bayesian information criterion (Schwarz, 1978) as implemented in jMODELTEST (Posada, 2008); TIM1 (Posada, 2003) and TIM2 (Posada, 2008), nucleotide substitution models with six free parameters and unequal base frequencies; TN (Tamura \& Nei, 1993), nucleotide substitution model with five free parameters and unequal base frequencies; K80 (Kimura, 1980), nucleotide substitution model with one free parameter and equal base frequencies; HKY (Hasegawa et al., 1985), nucleotide substitution model with four free parameters and unequal base frequencies; GTR (Tavaré, 1986), general time reversible model with eight free parameters; I, proportion of invariable sites; $G$, gamma rates; NS, not significant; NA, not applicable.

\begin{tabular}{|c|c|c|c|}
\hline \multirow[t]{2}{*}{ Parameters } & \multicolumn{3}{|c|}{ Phylogenetic marker } \\
\hline & cal1 & chi18-5 & tef1 \\
\hline No. of sequences & 51 & 50 & 51 \\
\hline No. of characters & 429 & 731 & 515 \\
\hline Parsimony informative & 31 & 32 & 49 \\
\hline Substitution models selected by AIC and BIC & $\mathrm{TIM} 2+\mathrm{G}$ and $\mathrm{K} 80+\mathrm{G}$ & $\mathrm{TIM} 1+\mathrm{G}$ and $\mathrm{HKY}+\mathrm{G}$ & $\operatorname{TrN}+\mathrm{G}$ and $\mathrm{K} 80$ \\
\hline MCMC generations, millions & 1 & 1 & 3 \\
\hline No. of chains/Temp. $(\lambda)$ & $4 / 0.2$ & $4 / 0.2$ & $4 / 0.2$ \\
\hline Sampling frequency & 100 & 100 & 100 \\
\hline 'Burned' & 300 & 300 & 300 \\
\hline \multicolumn{4}{|l|}{ Substitution rates ${ }^{\star}$} \\
\hline $\mathrm{A} \leftrightarrow \mathrm{T}$ & 0.23 & 0.11 & 0.30 \\
\hline $\mathrm{C} \leftrightarrow \mathrm{G}$ & 0.08 & 0.46 & 0.33 \\
\hline $\mathrm{C} \leftrightarrow \mathrm{T}$ & 80.60 & 7.11 & 0.51 \\
\hline $\mathrm{G} \leftrightarrow \mathrm{T}$ & 1 & 1 & 1 \\
\hline$\alpha^{*}$ & 0.06 & 0.06 & 0.08 \\
\hline \multirow[t]{2}{*}{ Total tree length ${ }^{*}$} & 7.05 & 5.09 & 6.73 \\
\hline & \multicolumn{3}{|c|}{ DNA polymorphism analysis } \\
\hline No. of sites excluding gaps and missing data & 377 & 395 & 445 \\
\hline Segregation sites & 32 & 34 & 57 \\
\hline No. of mutations, $\eta$ & 37 & 35 & 58 \\
\hline No. of alleles (haplotypes) & 11 & 9 & 15 \\
\hline
\end{tabular}

${ }^{\star}$ As estimated after GTR MCMC sampling and burning.

analysis of the plot showing generation versus the log probability of observing the data. According to the protocol of Leache \& Reeder (2002) PP values lower than 0.95 were not considered significant while values below 0.9 are not shown on the resulting phylograms. Model parameter summaries after MCMC runs and burning first samplings as well as nucleotide characteristics of the loci used are collected in Table 2.

Detection of recombination. Recombination within individual phylogenetic clades was tested by multiple tools. (a) The index of association $\left(I_{\mathrm{A}}\right)$, which measures whether the alleles from the different loci in a population are randomly or nonrandomly associated in the analysed genomes (Maynard Smith, 1992), and which was computed by using the linkage disequilibrium (LD) analysis available on the MLST website (http://linux.mlst.net/link_dis/index.htm); significance was gauged from 1000 random permutations of the data. (b) The partition homogeneity test (PHT) integrated in PAUP $^{\star} 4.0 \mathrm{~b} 10$ (Swofford, 2002), which estimates the congruence among different loci datasets (Cunningham, 1997). For this test heuristic search under the parsimony optimality criterion was used, parsimony-uninformative characters were excluded, gaps were treated as missing, and 10000 repetitions were performed. A maximum of 100 trees were saved to conserve memory. (c) Recombination tests implemented in the RecombiTEST package available at http://www.lifesci.sussex.ac. 
uk/CSE/test/. (d) The $\phi_{\mathrm{w}}$ test (pairwise homoplasy index, Phi) as implemented in the SplitsTree software (Huson, 1998). (e) Visual analysis of topologies of phylogenetic trees.

\section{RESULTS}

\section{Phylogenetic species in the sample of clinical Trichoderma strains}

Our sample consisted of 51 strains, all sharing the same sequence of ITS1 and 2 of the rRNA gene cluster. The sequence was identified as the ' $H$. orientalis- $T$. longibrachiatum' species doublet by the oligonucleotide barcode program TrichOKey (Druzhinina et al., 2005; online at http://www.ISTH.info). Among these strains, 15 were obtained as clinical isolates. The other 36 were chosen to cover the broadest possible geographical variability of $T$. longibrachiatum including all available strains (7) of its putative teleomorph $\mathrm{H}$. orientalis. In order to investigate the evolutionary relations within this sample, we sequenced three phylogenetic markers used in Hypocrea/Trichoderma: the long intron of tef1, an intron-containing fragment of call and a partial exon sequence of chil8-5. Nucleotide characteristics of these sequences are given in Table 2 .

We used Bayesian analysis of the individual gene datasets to infer a phylogenetic structure (Fig. 1a-c). Three statistically supported clades and a single lone branch were present in the tef1 and chil8-5 gene trees (Fig. 1a, b): the PS (phylogenetic species) I clade contained more than half the isolates, including the ex-type strain of T. longibrachiatum; clade PS II included the ex-type strain of $H$. orientalis, all but one (C.P.K. 1261) of the other strains of H. orientalis, and three strains originally identified as T. longibrachiatum; clade PS III contained three strains isolated from coffee rhizosphere in Ethiopia. The single strain isolated from the teleomorph from New Zealand (C.P.K. 1261) formed a branch with unresolved phylogenetic position within the dataset studied. Resolution of the call tree (Fig. 1c) was less clear as no statistical support was detected for clade PS I, but its topology was in agreement with the other two loci. The phylogenetic position of strains forming clade PS II in tef1 and chi18-5 trees was not well resolved by call analysis as C.P.K. 2880 and C.P.K. 2881 formed a topologically separated subclade (but without statistical support) from other strains of PS II. Positions of C.P.K. 1261 and PS III on the cal1 tree were concordant with the other two loci.

This analysis shows that the three clades and the single branch are fully supported in two gene trees, and not rejected in the third. Thus, at least three clades (PS I, II and III) fulfil the two criteria of Dettman et al. (2003) for multilocus genealogies - i.e. that a clade must be present in the majority of single-locus trees; and that a clade is reliably supported by at least one single-locus genealogy and is not contradicted by any other single-gene tree determined by the same methods (the genealogical nondiscordance criterion). Based on the position of the ex-type strains for T. longibrachiatum and $H$. orientalis in different clades, we conclude that clade PS I represents T. longibrachiatum sensu stricto, and clade PS II consequently represents $H$. orientalis. On the other hand, clade PS III and C.P.K. 1261 are two as yet undescribed species of section Longibrachiatum which we will further refer to as Trichoderma sp. PS (phylogenetic species) III and Hypocrea sp. PS IV, respectively.

The clinical strains of ' $T$. longibrachiatum' were only found in clades PS I (12 strains) and II (3 strains) and thus - in contrast to what was believed before - belong to two phylogenetic species (Fig. 1).

\section{Reproductive strategies of $T$. longibrachiatum and $\boldsymbol{H}$. orientalis}

In order to test whether the phylogenetically distinct species $T$. longibrachiatum and $H$. orientalis are also separated by a reproductive barrier, we used the split decomposition method provided by the SplitsTree (Huson \& Bryant, 2006) package. This analysis enabled us to test for the presence of network relationships within clades PS I, II and III, and various dual and threefold combinations of them, using a concatenated dataset of tef1, call and chit18-5 (Fig. $2 \mathrm{a}-\mathrm{c})$. This method presents conflicting phylogenetic data, presumably arising from recombination, as an interconnected network of lineages. As shown in Fig. 2(a), such a network was evident between the total dataset (four phylogenetic species). However, analyses of individual clades PS I and PS II documented an almost complete lack of a network for T. longibrachiatum compared to $H$. orientalis, which is expected to have sexual reproduction in its life cycle as several teleomorphs were collected. When the Phi test (Huson \& Bryant, 2006) implemented in the same software package was applied to various concatenated gene combinations of clades PS I, PS II and PS III, evidence for recombination was obtained for every combination (data not shown) and also for clade PS II (H. orientalis) alone $(\phi=0.32, P=0.0005)$, whereas the possibility of recombination was rejected for clade PS I ( $T$. longibrachiatum) $(\phi=0.14$, $P=0.58$ ). These data indicate that in contrast to $H$. orientalis, which represents a sexual population, $T$. longibrachiatum is largely clonal. Strains of PS III could not be analysed individually because they were too low in number.

The partition homogeneity test (PHT; Huelsenbeck et al., 1996) was used to examine the congruence between gene trees. In this test artificial datasets are produced by multiple (10000) resampling and random swapping of observed datasets and subsequent construction of maximum-parsimony trees for every newly sampled 'gene' sequence. For clonally reproducing populations, the sums of the lengths of the gene trees for the observed and resampled data should be similar, but under recombination the sums of the tree lengths should be longer than that for the actual data because recombination among unlinked sites should introduce homoplasy into the data. When the whole dataset was analysed (PS I-IV; call, chi18-5 and tef1) the 
(a)

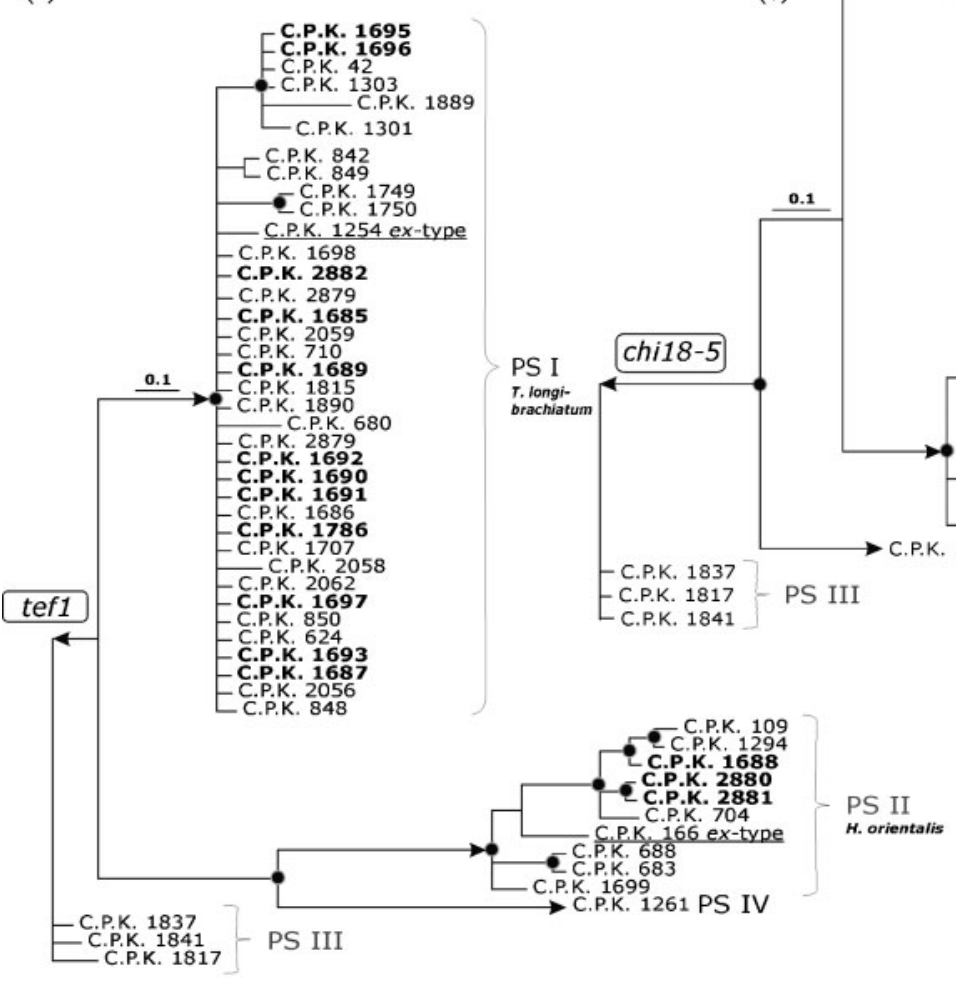

C.P.K. 1749 C.P.K. 710 C.P.K. 1685 - C.P.K. 2056 C.P.K. 2058 C.P.K. 1686 C.P.K. 1698 C.P. 42 C.P.K. 848 PS I

\begin{tabular}{l|l} 
C.P.K. 842 \\
C.P.K. 45 & $\begin{array}{l}\text { T. rongi- } \\
\text { brachiatum }\end{array}$ \\
\hline -C.P.K. 244 &
\end{tabular}

(c) -C.P.K. 1707 - C.P.K. 2882 C.P.K. 2879 C.P.K. 1889 -C.P.K. 1687 C.P.K. 1692 - C.P.K. 1303 -C.P.K. 849 C.P.K. 849

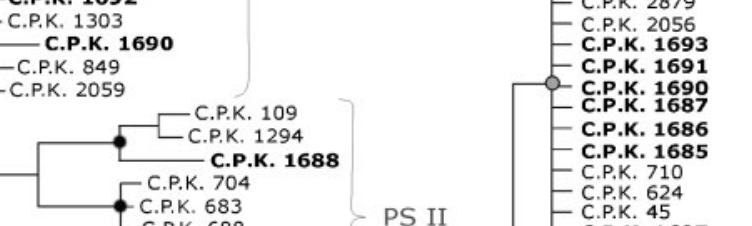
C.P.K. 850 - C.P.K. 842 - C.P.K. 2062 - C.P.K. 2059 C.P.K. 2058 C C.P.K. 84 C.P.K. 2879 C.P.K. 1693 C.P.K. 1686 C.P.K. 710 - C.P.K. 624 C.P.K. 683
C.P.K. 68

C.P.K. 2880 C.P.K. 2881 C.P.K. 166 ex-type

88

\section{E C.P.K. 1697
0.1 \\ C.P.K. 28
K. 1696}

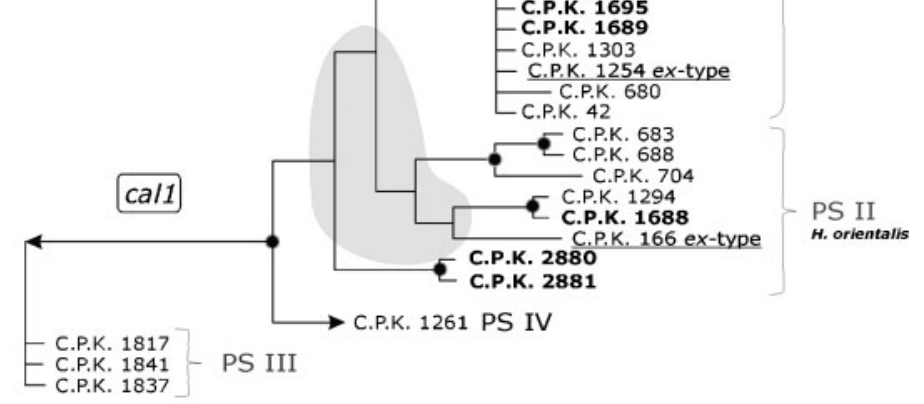

Fig. 1. Bayesian phylogenetic analysis of clinical Trichoderma strains (in bold) and environmental isolates of $T$. longibrachiatum and $H$. orientalis based on partial sequences of tef1 (a), chi18-5 (b) and cal1 (c). Nodes marked by black circles indicate posterior probabilities (PP) $>0.94$; nodes marked by grey circles indicate $0.95>$ PP $>0.90$. Arrows mark branches leading to recognized phylogenetic species. Ex-type strains of $T$. longibrachiatum and $H$. orientalis are underlined. The shadowing indicates an unresolved area on the cal1 phylogram. 
(a)

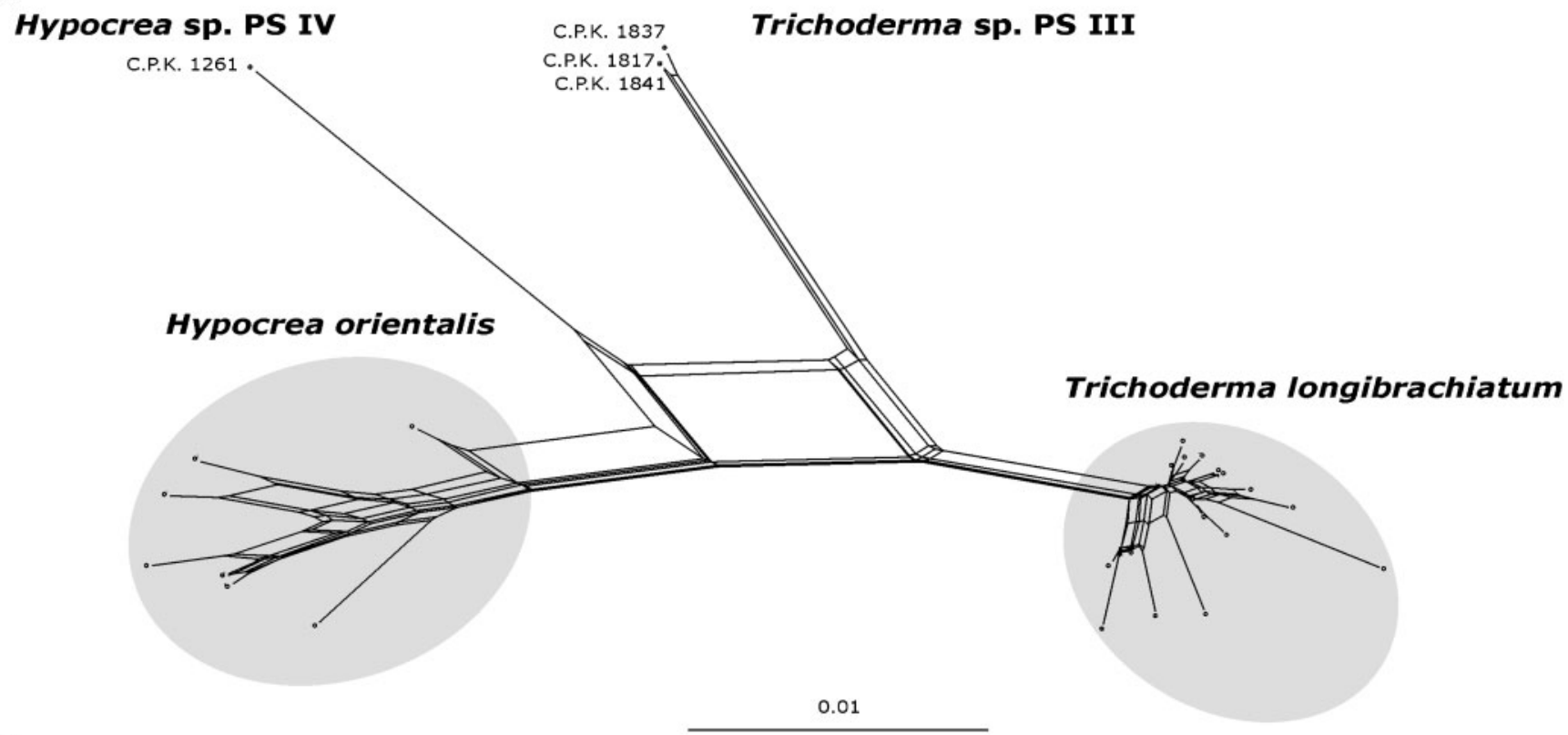

(b)

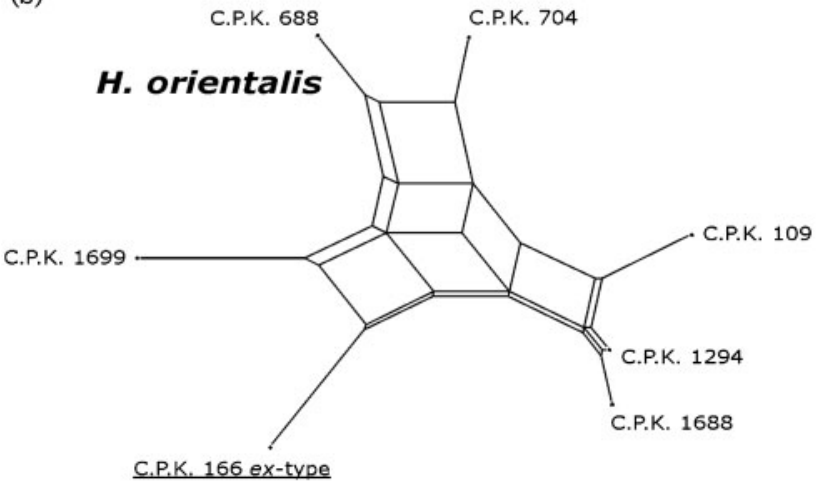

(c)

\section{T. longibrachiatum}

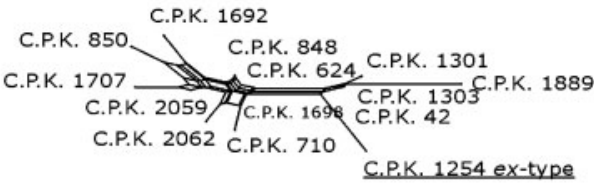

Fig. 2. Reconstruction of possible recombination networks between and within $T$. longibrachiatum and $H$. orientalis by the split decomposition method applied to the concatenated dataset (tef1+cal1+chi18-5). (a) Total sample with four phylogenetic species; (b) strains of $H$. orientalis clade PS II; (c) strains of $T$. longibrachiatum clade PS I. Gaps were treated as missing characters throughout. All networks have been calibrated to fit one scale.

actual summed tree length of 189 steps was exactly at the lowest limit of that produced by any of the 10000 artificial datasets $(P=0.0003)$, and eight steps shorter than $0.95 \%$ of them, thus indicating incongruence among the different gene trees. In order to test whether this topological conflict appears to be due to incongruence in one particular clade the PHT was applied to the PS I and PS II clades separately. In addition, we ran the analysis with and without partition of the tefl gene, which covers the intron sequence and therefore may contain homoplasious characters even in clonal populations, due to high mutation rates. The corresponding data show (Fig. 3) that there is a recombination within the PS II $H$. orientalis clade, the result being independent of tef 1 sequences. At the same time, topologies of call and chils-5 trees for PS I T. longibrachiatum are congruent, suggesting the absence of sexual recombination in this clade (Fig. 3). When tef1 was included in the PHT of PS I the null hypothesis of recombination was not rejected $(P=0.046)$.

In order to verify this result by yet further means, we also used the index of association (IA) test on a subset of 'clone corrected' data (i.e. individuals with identical genotypes at the three loci were excluded so that each multilocus genotype was represented only once; cf. Pringle et al., 2005). The data obtained were in accordance with occurrence of recombination within $H$. orientalis but not in T. longibrachiatum and Trichoderma sp. PS III, as the IA test did not reject the null hypothesis of recombination in the former $(P=0.462)$ but did so in the latter two datasets.

Finally, the maximum chi-squared test of Maynard Smith (1992), linkage disequilibrium (LD) r2 (Hill \& Robertson, 


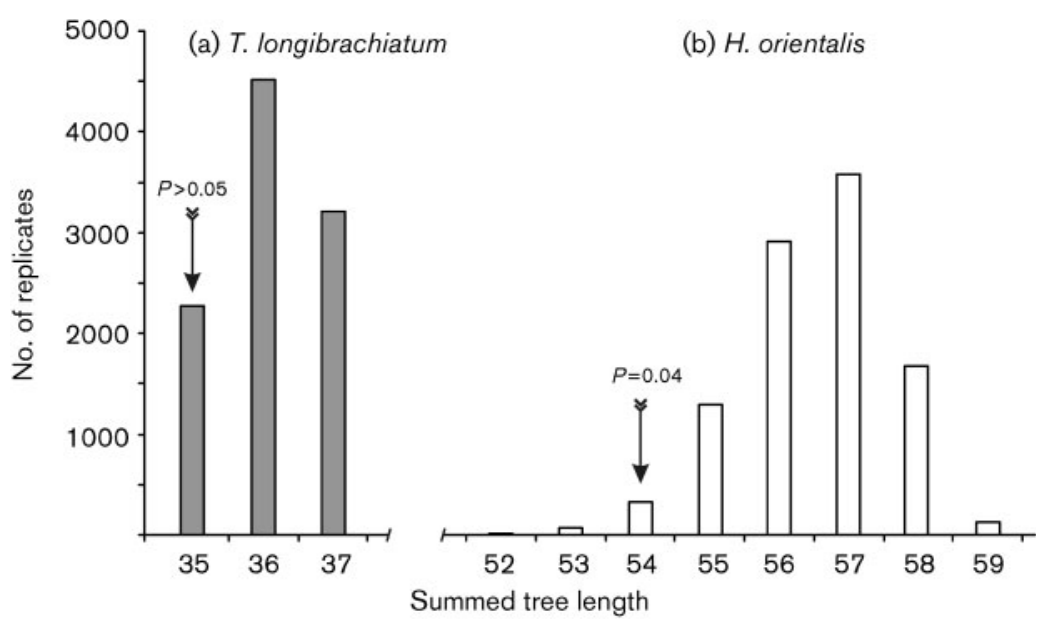

Fig. 3. PHT results for $T$. longibrachiatum (a) and $H$. orientalis (b) applied to cal1 and chi185 sequences. Arrows indicate the actual summed tree length and corresponding $P$ value. For the test parsimony-uninformative characters were excluded, gaps were treated as missing characters, and 10000 repetitions of heuristic search under parsimony criterion were performed.
1968), and LDD' (LD versus distance IDI; Lewontin, 1964) estimates available on the RecombiTEST webpage (see Methods) also detected recombination between strains of $H$. orientalis, but not in $T$. longibrachiatum (data not shown).

Inspection of intracladal structures of $H$. orientalis on all trees revealed several conflicts of topologies. Besides the abovementioned unresolved position of C.P.K. 2880 and C.P.K. 2881 on the call tree, other strains of PS II aggregated in different statistically supported subclades which were unique for every locus. Such 'jumping' behaviour of individual sequences also suggests the presence of sexual recombination. No intracladal patterns were detected for PS I and III.

\section{Haplotype structure and distribution of clinical strains within the $T$. longibrachiatum clade}

Since the above data showed that T. longibrachiatum behaves essentially clonally, we now tested whether the clinical isolates would occupy specific positions in the structure of clade PS I, i.e. whether clinical occurrence would correlate with specific haplotypes. To this end, we used DnaSP 4.50.3 to collapse individual sequence alignments to haplotypes, and then subjected them to statistical parsimony implemented in TCS (Clement et al., 2000). The results showed that the haplotype with the highest total numbers of environmental isolates also contained the majority of clinical isolates. Haplotypes represented by only a single strain contained only a few individual clinical isolates, but most of them were represented by wild-type strains (data not shown).

\section{Biogeography of $\boldsymbol{T}$. longibrachiatum and H. orientalis}

The distribution analysis of strains from PS I and II shows that $T$. longibrachiatum and $H$. orientalis are a closely related pair of cosmopolitan and sympatric species, as both of them were detected on almost all continents. H. orientalis was frequently isolated from soil or mud samples as the anamorph, so it is clearly a holomorphic species which may coexist with $T$. longibrachiatum in the same ecosystem. The only known teleomorph samples of $H$. orientalis have so far been collected in China, thus making it difficult to speculate on climatic preferences for fruit body formation. Unfortunately the origin of several clinical isolates (6; Table 1) was not available for this study. The other strains were isolated from hospitals in North America (south United States and north-east Canada), temperate Europe (central France and Austria), subtropical Gran Canaria and continental Spain. This dataset shows that there is no bias of clinical strains towards specific geographical location.

\section{DISCUSSION}

This study set out to test the hypothesis that opportunistic strains of $T$. longibrachiatum may represent specialized potentially clonal subpopulations within this species. In that case it would be possible to identify specific genetic markers for their diagnosis. Availability of such sequences would enable screening for the source of these infections and so eventually reduce their clinical appearance. In contrast, the results from this work provide clear evidence that not only one but two genetically different species $T$. longibrachiatum and $H$. orientalis infect immunocompromised patients, and that clinical isolates are found in all gene haplotypes or are predominantly associated with the major haplotypes of both species. This leads us to conclude that not a single population of $T$. longibrachiatum is responsible for opportunistic attack on humans but presumably every isolate of $T$. longibrachiatum or $H$. orientalis is potentially able to do so. This conclusion is also supported by phenotype microarray data testing carbon source utilization profiles of these two species, which failed to show any statistically supported metabolic difference (L. Hatvani, L. Kredics \& I. S. Druzhinina, unpublished). It is further consistent with data obtained from isoenzyme analysis (Szekeres et al., 2006) and study of mitochondrial 
DNA polymorphism (Antal et al., 2006). We have thus demonstrated that both species are a potential threat. It is important to mention that human clinical isolates shared identical multilocus haplotypes with isolates from soil and plant materials, and Trichoderma invasive mycoses may therefore be potentially nosocomial.

The finding of this work has important implications also for the biotechnological use of $T$. longibrachiatum, because strains of this fungus have been used as agents of biological control against phytopathogenic fungi (Vizcaíno et al., 2005; Sánchez et al., 2007). In the light of the present results, this application should be abandoned or at least carefully monitored. Also, T. longibrachiatum has been reported to be a component of the indoor fungal flora (Thrane et al., 2001) and has also frequently been isolated from mushroom farms infected by green mould disease (Hatvani et al., 2007). We recommend that T. longibrachiatum and $H$. orientalis are included on the list of those indoor fungi whose presence is specifically monitored. Primers to be designed for such tests therefore have to take into account the whole genetic variation within $T$. longibrachiatum and $H$. orientalis, as demonstrated in this paper. The lower percentage of $H$. orientalis clinical strains may be a result of the lower sample size. Interestingly, clinical isolates in the $H$. orientalis clade were recovered during the last 5 years, whereas isolates recovered earlier were only T. longibrachiatum.

There have so far been no studies dedicated to the ecology of $T$. longibrachiatum and $H$. orientalis. Therefore it is difficult to trace their preferred ecological niches, which would aid prediction of the source of Trichoderma infections. Nevertheless, besides being isolated from numerous soil samples worldwide, $T$. longibrachiatum has been consistently detected in association either with wild fruiting bodies of the wood-decaying fungus Pleurotus ostreatus (L. Hatvani, L. Kredics, I. S. Druzhinina \& C. P. Kubicek, unpublished) or in mushroom farms cultivating Pleurotus and Agaricus (Hatvani et al., 2007). Another also interesting case of abundant detection of $T$. longibrachiatum was its isolation from the archaeological excavation sites at an Iron Age tomb in the Republic of Tatarstan, Russia (F. Alimova \& I. S. Druzhinina, unpublished). An explanation for these findings may be derived from observations that $T$. longibrachiatum occupies the lowest soil horizons but not the upper organic soil layers. Therefore, together with its appearance as causative agent of invasive mycoses, there is emerging evidence that $T$. longibrachiatum and $H$. orientalis may have a specialized ecological niche(s) which is(are) essentially different from other species of the genus.

The present findings distinguish infections caused by $T$. longibrachiatum and $H$. orientalis from those caused by human-pathogenic fungi from the Fusarium solani complex, where the majority of clinical isolates are derived from a single worldwide distributed clonal lineage (Zhang et al., 2006). A clonal structure has also been demonstrated for other pathogenic fungi such as Trichophyton rubrum, Cryptococcus neoformans or the chytrid pathogen Batrachochytrium dendrobatidis (Gräser et al., 1999; Halliday \& Carter, 2003; Morehouse et al., 2003), whereas many other human-pathogenic fungi, including Aspergillus fumigatus (Pringle et al., 2005), exhibit both clonal and recombining strategies (for reviews see Taylor et al., 1999; Nielsen \& Heitman, 2007). The results from this study present evidence that sexual reproduction, indicated by recombination, is an important strategy in one of the two opportunistic pathogenic species of Hypocrea/Trichoderma, $H$. orientalis. The potential presence of a sexual life cycle in an opportunistic pathogen is a significant finding, because its allows the fungus to respond faster to environmental challenges, thereby combating disease treatment by exchange of antibiotic-resistance genes and virulence factors (Milgroom, 1996; Nielsen \& Heitman, 2007; Normak et al., 2003; Paoletti et al., 2005). It is not known whether these two species exploit their animal-pathogenic ability in nature; therefore it is too early to speculate that $H$. orientalis has developed different strategies than $T$. longibrachiatum for its opportunistic attack and subsequent survival. The phylogenetic analysis presented in this paper suggests that $T$. longibrachiatum and $H$. orientalis evolved in parallel from a common ancestor, forming two sympatric species. Thus, their pathogenic ability would be the result of a heritage from a recent ancestor rather than a convergent evolution. Further studies are needed to understand which species exploits more of its pathogenic abilities in nature.

H. orientalis is not the only Hypocrea species with potential health risk to humans. Druzhinina et al. (2008) reported a case study in which Hypocrea sp. CBS 120951 was isolated from the lung tissue of a patient with non-fatal pulmonary fibrosis. This isolate exhibits an uncertain phylogenetic position in the genus Hypocrea/Trichoderma and is also phenotypically very distinct as it does not conidiate in vitro but produces fertile stromata. Nevertheless, cases of sexual reproduction among clinically relevant strains of Hypocrea may be more frequent than was previously recognized.

Apart from these clinical implications, the results presented here also provide some new insights into the taxonomy of $T$. longibrachiatum and $H$. orientalis: based on ITS1 sequence analysis and isoenzyme data, Samuels et al. (1998) suggested that $T$. longibrachiatum may be the anamorph of $H$. orientalis. The present data reject this hypothesis by clearly showing that these two taxa represent individual phylogenetic species, which have already undergone reproductive isolation. In addition, we provide evidence for a third phylogenetic species, Trichoderma sp. PS III, which should be formally described elsewhere. In addition, the branches leading to this species did not form a network in the split decomposition analysis although the three available strains were isolated from three different coffee-growing areas in Ethiopia. These data are supportive of a reproductive barrier between Trichoderma sp. PS III, T. longibrachiatum and $H$. orientalis. 
Hypocrea sp. strain C.P.K. 1261 (=CBS 243.63) formed a separate branch in all gene trees and in the SplitsTree analysis. Samuels et al. (1998) had previously noted that this strain differed from the other isolates in their sample both morphologically and in isoenzyme analysis profiles but nevertheless maintained it within their concept of $H$. orientalis. Based on our data, this strain represents a fourth species in our dataset. Since the origin of this strain was from a fruiting body, it is a member of another sexually propagating population which could occupy an as yet unknown ecological niche.

\section{ACKNOWLEDGEMENTS}

This work was supported partly by Austrian Science Fund grant FWF P-19340-MOB to C.P. K., partly by Hungarian Scientific Research Fund grant F68381 to L. K.; L. K. is grantee of the János Bolyai Research Scholarship (Hungarian Academy of Sciences). The authors thank John Bissett, Ilona Dóczi, Mirja Salkinoja-Salonen and George Szakacs for the gift of some of the strains. We also acknowledge Katarzyna Szymanska for her help in laboratory work.

\section{REFERENCES}

Akaike, H. (1974). A new look at the statistical model identification. IEEE Trans Automat Contr 19, 716-723.

Antal, Z., Varga, J., Kredics, L., Szekeres, A., Hatvani, L., Manczinger, L., Vágvölgyi, C. \& Nagy, E. (2006). Intraspecific mitochondrial DNA polymorphism within the emerging filamentous fungal pathogen Trichoderma longibrachiatum. J Med Microbiol 55, 31-35.

Chaverri, P., Castlebury, L. A., Samuels, G. J. \& Geiser, D. M. (2003). Multilocus phylogenetic structure within the Trichoderma harzianum/ Hypocrea lixii complex. Mol Phylogenet Evol 27, 302-313.

Chouaki, T., Lavarde, V., Lachaud, L., Raccurt, C. P. \& Hennequin, C. (2002). Invasive infections due to Trichoderma species: report of 2 cases, findings of in vitro susceptibility testing, and review of the literature. Clin Infect Dis 35, 1360-1367.

Clement, M., Posada, D. \& Crandall, K. (2000). TCS: a computer program to estimate gene genealogies. Mol Ecol 9, 1657-1660.

Cunningham, C. W. (1997). Is incongruence between data partitions a reliable predictor of phylogenetic accuracy? Empirical testing an iterative procedure for choosing among phylogenetic methods. Syst Biol 46, 464-478.

Dettman, J. R., Jacobson, D. J. \& Taylor, J. W. (2003). A multilocus genealogical approach to phylogenetic species recognition in the model eukaryote Neurospora. Evolution 57, 2703-2720.

Druzhinina, I. S., Kopchinskiy, A. G., Komon, M., Bissett, J., Szakacs, G. \& Kubicek, C. P. (2005). An oligonucleotide barcode for species identification in Trichoderma and Hypocrea. Fungal Genet Biol 42, 813-828.

Druzhinina, I. S., LaFe, K., Willinger, B., Komoń-Zelazowska, M., Ammirati, J., Kubicek, C. P. \& Rogers, J. D. (2008). An unknown species from Hypocreaceae isolated from human lung tissue of a patient with non-fatal pulmonary fibrosis. Clin Microbiol Newsl 29, 180-184.

Furukawa, H., Kusne, S., Sutton, D. A., Manez, R., Carrau, R., Nichols, L., Abu-Elmagd, K., Skedros, D., Todo, D. \& Rinaldi, M. G. (1998). Acute invasive sinusitis due to Trichoderma longibrachiatum in a liver and small bowel transplant recipient. Clin Infect Dis 26, 487-489.
Gräser, Y., Kühnisch, J. \& Presber, W. (1999). Molecular markers reveal exclusively clonal reproduction in Trichophyton rubrum. J Clin Microbiol 37, 3713-3717.

Halliday, C. L. \& Carter, D. A. (2003). Clonal reproduction and limited dispersal in an environmental population of Cryptococcus neoformans var. gattii isolates from Australia. J Clin Microbiol 41, 703-711.

Harman, G. E., Howell, C. R., Viterbo, A., Chet, I. \& Lorito, M. (2004). Trichoderma species - opportunistic, avirulent plant symbionts. Nat Rev Microbiol 2, 43-56.

Hasegawa, M., Kishino, K. \& Yano, T. (1985). Dating the human-ape splitting by a molecular clock of mitochondrial DNA. J Mol Evol 22, $160-174$.

Hatvani, L., Antal, Z., Manczinger, L., Szekeres, A., Druzhinina, I. S., Kubicek, C. P., Nagy, A., Nagy, E., Vágvölgyi, C. \& Kredics, L. (2007). Green mold diseases of Agaricus and Pleurotus spp. are caused by related but phylogenetically different Trichoderma species. Phytopathology 97, 532-537.

Hennequin, C., Chouaki, T., Pichon, J. C., Strunski, V. \& Raccurt, C. (2000). Otitis externa due to Trichoderma longibrachiatum. Eur J Clin Microbiol Infect Dis 19, 641-642.

Hill, W. G. \& Robertson, A. (1968). Linkage disequilibrium in finite populations. Theor Appl Genet 38, 226-231.

Huelsenbeck, J. P., Bull, J. J. \& Cunningham, C. W. (1996). Combining data in phylogenetic analysis. Trends Ecol Evol 11, 152158.

Huson, D. H. (1998). SplitsTree: a program for analyzing and visualizing evolutionary data. Bioinformatics 14, 68-73.

Huson, D. H. \& Bryant, D. (2006). Application of phylogenetic networks in evolutionary studies. Mol Biol Evol 23, 254-267.

Jaklitsch, W. M., Komon, M., Kubicek, C. P. \& Druzhinina, I. S. (2006). Hypocrea crystalligena sp. nov., a common European species with a white-spored Trichoderma anamorph. Mycologia 98, 499-513.

Kimura, M. (1980). A simple method for estimating evolutionary rate of base substitutions through comparative studies of nucleotide sequences. J Mol Evol 16, 111-120.

Klein, D. \& Eveleigh, D. E. (1998). Ecology of Trichoderma. Trichoderma and Gliocladium, vol. 1, Basic Biology, Taxonomy and Genetics, pp. 57-74. Edited by C. P. Kubicek \& G. E. Harman. London, UK: Taylor \& Francis.

Komoń-Zelazowska, M., Bissett, J., Zafari, D., Hatvani, L., Manczinger, L., Woo, S., Lorito, M., Kredics, L., Kubicek, C. P. \& Druzhinina, I. S. (2007). Genetically closely related but phenotypically divergent Trichoderma species cause world-wide green mould disease in oyster mushroom farms. Appl Environ Microbiol 73, 7415-7426.

Kratzer, C., Tobudic, S., Schmoll, M., Graninger, W. \& Georgopoulos, A. (2006). In vitro activity and synergism of amphotericin B, azoles and cationic antimicrobials against the emerging pathogen Trichoderma spp. J Antimicrob Chemother 58, 1058-1061.

Kredics, L., Antal, Z., Dóczi, I., Manczinger, L., Kevei, F. \& Nagy, E. (2003). Clinical importance of the genus Trichoderma. A review. Acta Microbiol Immunol Hung 50, 105-117.

Kubicek, C. P., Bissett, J., Kullnig-Gradinger, C. M., Druzhinina, I. S. \& Szakacs, G. (2003). Genetic and metabolic diversity of Trichoderma: a case study on South-East Asian isolates. Fungal Genet Biol 38, 310-317.

Kuhls, K., Lieckfeldt, E., Samuels, G. J., Meyer, W., Kubicek, C. P. \& Börner, T. (1997). Revision of Trichoderma sect. Longibrachiatum including related teleomorphs based on analysis of ribosomal DNA internal transcribed spacer sequences. Mycologia 89, 442-460.

Kuhls, K., Lieckfeldt, E., Börner, T. \& Guého, E. (1999). Molecular re-identification of human pathogenic Trichoderma isolates as 
Trichoderma longibrachiatum and Trichoderma citrinoviride. Med Mycol 37, 25-33.

Kullnig, C. M., Szakacs, G. \& Kubicek, C. P. (2000). Molecular identification of Trichoderma species from Russia, Siberia and the Himalaya. Mycol Res 104, 1117-1125.

Leache, A. D. \& Reeder, T. W. (2002). Molecular systematics of the eastern fence lizard (Sceloporus undulatus): a comparison of parsimony, likelihood and Bayesian approaches. Syst Biol 51, 44-68.

Lewontin, R. C. (1964). The interaction of selection and linkage. I. General considerations: heterozygotic models. Genetics 49, 49-67.

Maynard Smith, J. (1992). Analyzing the mosaic structure of genes. $J$ Mol Evol 34, 126-129.

Milgroom, M. G. (1996). Recombination and the multilocus structure of fungal populations. Annu Rev Phytopathol 34, 457-477.

Morehouse, E. A., James, T. Y., Ganley, A. R. D., Vilgalys, R., Berger, D., Murphy, P. J. \& Longcore, J. E. (2003). Multilocus sequence typing suggests the chytrid pathogen of amphibians is a recently emerged clone. Mol Ecol 12, 395-403.

Munoz, F. M., Demmler, G. J., Travis, W. R., Ogden, A. K., Rossmann, S. N. \& Rinaldi, M. G. (1997). Trichoderma longibrachiatum infection in a pediatric patient with aplastic anemia. J Clin Microbiol 35, 499-503.

Myoken, Y., Sugata, T., Fujita, Y., Asaoku, H., Fujihara, M. \& Mikami, Y. (2002). Fatal necrotizing stomatitis due to Trichoderma longibrachiatum in a neutropenic patient with malignant lymphoma: a case report. Int J Oral Maxillofac Surg 31, 688-691.

Nicholas, K. B. \& Nicholas, H. B., Jr (1997). Genedoc: a tool for editing and annotating multiple sequence alignments. http:// www.psc.edu/biomed/genedoc.

Nielsen, K. \& Heitman, J. (2007). Sex and virulence of human pathogenic fungi. Adv Genet 57, 143-173.

Normak, B. B., Judson, O. P. \& Morgan, N. A. (2003). Genomic signatures of ancient asexual lineages. Biol J Linn Soc Lond 79, 69-84.

Paoletti, M., Rydholm, C., Schwier, E. U., Anderson, M. J., Szakacs, G., Lutzoni, F., Debeaupuis, J. P., Latgé, J. P., Denning, D. W. \& other authors (2005). Evidence for sexuality in the opportunistic fungal pathogen Aspergillus fumigatus. Curr Biol 15, 1242-1248.

Posada, D. (2003). Using Modeltest and PAUP* to select a model of nucleotide substitution. In Current Protocols in Bioinformatics, pp. 6.5.1-6.5.14. Edited by A. D. Baxevanis, D. B. Davison, R. D. M. Page, G. A. Petsko, L. D. Stein \& G. D. Stormo. New York: Wiley.

Posada, D. (2008). jModelTest: Phylogenetic Model Averaging. Mol Biol Evol (in press).doi:10.1093/molbev/msn083.

Pringle, A., Baker, D. M., Platt, J. L., Wares, J. P., Latge, J. P. \& Taylor, J. W. (2005). Cryptic speciation in the cosmopolitan and clonal human pathogenic fungus Aspergillus fumigatus. Evolution 59, 1886-1899.

Richter, S., Cormican, M. G., Pfaller, M. A., Lee, C. K., Gingrich, R., Rinaldi, M. G. \& Sutton, D. A. (1999). Fatal disseminated Trichoderma longibrachiatum infection in an adult bone marrow transplant patient: species identification and review of the literature. J Clin Microbiol 37, 1154-1160.

Ronquist, F. \& Huelsenbeck, J. P. (2003). MRBAYES 3: Bayesian phylogenetic inference under mixed models. Bioinformatics 19, 1572-1574.

Rozas, J., Sanchez-DelBarrio, J. C., Messeguer, X. \& Rozas, R. (2003). DnaSP, DNA polymorphism analyses by the coalescent and other methods. Bioinformatics 19, 2496-2497.

Samuels, G. J., Petrini, O., Kuhls, K., Lieckfeldt, E. \& Kubicek, C. P. (1998). The Hypocrea schweinitzii complex and Trichoderma sect. Longibrachiatum. Stud Mycol 41, 1-54.

Sánchez, V., Rebolledo, O., Picaso, R. M., Cárdenas, E., Córdova, C., González, O. \& Samuels, G. J. (2007). In vitro antagonism of
Thielaviopsis paradoxa by Trichoderma longibrachiatum. Mycopathologia 163, 49-58.

Schwarz, G. (1978). Estimating the dimension of a model. Ann Stat 6, 461-464.

Seguin, P., Degeilh, B., Grulois, G., Gacouin, A., Maugendre, S., Dufour, T., Dupont, B. \& Camus, C. (1995). Successful treatment of a brain abscess due to Trichoderma longibrachiatum after surgical resection. Eur J Clin Microbiol Infect Dis 14, 445-448.

Swofford, D. L. (2002). PAUP*: Phylogenetic Analysis Using Parsimony (*and Other Methods), Version 4.0b10. Sinauer Associates, Sunderland, MA.

Szekeres, A., Láday, M., Kredics, L., Varga, J., Antal, Z., Hatvani, L., Manczinger, L., Vágvölgyi, C. \& Nagy, E. (2006). Rapid identification of clinical Trichoderma longibrachiatum isolates by cellulose-acetate electrophoresis-mediated isoenzyme analysis. Clin Microbiol Infect 12, 369-375.

Tamura, K. \& Nei, M. (1993). Estimation of the number of nucleotide substitutions in the control region of mitochondrial DNA in humans and chimpanzees. Mol Biol Evol 10, 512-526.

Tang, P., Mohan, S., Sigler, L., Witterick, I., Summerbell, R., Campbell, I. \& Mazzulli, T. (2003). Allergic fungal sinusitis associated with Trichoderma longibrachiatum. J Clin Microbiol 41, 5333-5336.

Tanis, B. C., van der Pijl, H., van Ogtrop, M. L., Kibbelaar, R. E. \& Chang, P. C. (1995). Fatal fungal peritonitis by Trichoderma longibrachiatum complicating peritoneal dialysis. Nephrol Dial Transplant 10, 114-116.

Tavaré, S. (1986). Some probabilistic and statistical problems in the analysis of DNA sequences. In Some Mathematical Questions in Biology - DNA Sequence Analysis, pp. 57-86. Edited by R. M. Miura. Providence, RI: American Mathematical Society.

Taylor, J. W., Geiser, D. M., Burt, A. \& Koufopanou, V. (1999). The evolutionary biology and population genetics underlying fungal strain typing. Clin Microbiol Rev 12, 126-146.

Thompson, J. D., Gibson, T. J., Plewniak, F., Jeanmougin, F. \& Higgins, D. G. (1997). The CLUSTAL_X Windows interface: flexible strategies for multiple sequence alignment aided by quality analysis tools. Nucleic Acids Res 25, 4876-4882.

Thrane, U., Poulsen, S. B., Nirenberg, H. I. \& Lieckfeldt, E. (2001). Identification of Trichoderma strains by image analysis of HPLC chromatograms. FEMS Microbiol Lett 203, 249-255.

Turner, D., Kovacs, W., Kuhls, K., Lieckfeldt, E., Peter, B., ArisanAtac, I., Strauss, J., Samuels, G. J., Börner, T. \& Kubicek, C. P. (1997). Biogeography and phenotypic variation in Trichoderma sect. Longibrachiatum and associated Hypocrea species. Mycol Res 101, 449-459.

Vizcaíno, J. A., Sanz, L., Basilio, A., Vicente, F., Gutiérrez, F., Hermosa, M. R. \& Monte, E. (2005). Screening of antimicrobial activities in Trichoderma isolates representing three Trichoderma sections. Mycol Res 109, 1397-1406.

Walsh, T. J. \& Groll, A. H. (1999). Emerging fungal pathogens: evolving challenges to immunocompromised patients for the twenty-first century. Transpl Infect Dis 1, 247-261.

Wilgenbusch, J. C., Warren, D. L. \& Swofford, D. L. (2004). AWTY: a system for graphical exploration of MCMC convergence in Bayesian phylogenetic inference. http://ceb.csit.fsu.edu/awty.

Wuczkowski, M., Druzhinina, I. S., Gherbawy, Y., Klug, B., Prillinger, H. J. \& Kubicek, C. P. (2003). Species pattern and genetic diversity of Trichoderma in a mid-European, primeval floodplain-forest. Microbiol Res 158, 125-133.

Zhang, C. L., Druzhinina, I. S., Kubicek, C. P. \& Xu, T. (2005). Biodiversity of Trichoderma in China: evidence for a North to South 
difference of species distribution in East Asia. FEMS Microbiol Lett 251, 251-257.

Zhang, N., O'Donnell, K., Sutton, D. A., Nalim, F. A., Summerbell, R. C.,

Padhye, A. A. \& Geiser, D. M. (2006). Members of the Fusarium solani species complex that cause infections in both humans and plants are common in the environment. J Clin Microbiol 44, 2186-2190.

Edited by: H. A. B. Wösten 